\title{
Single-source chip-based frequency comb enabling extreme parallel data transmission
}

Hu, Hao; Da Ros, Francesco; Pu, Minhao; Ye, Feihong; Ingerslev, Kasper; Porto da Silva, Edson; Nooruzzaman, Md; Amma, Yoshimichi; Sasaki, Yusuke; Mizuno, Takayuki

Total number of authors:

19

Published in:

Nature Photonics

Link to article, DOI:

$10.1038 / \mathrm{s} 41566-018-0205-5$

Publication date:

2018

Document Version

Peer reviewed version

Link back to DTU Orbit

Citation $(A P A)$ :

Hu, H., Da Ros, F., Pu, M., Ye, F., Ingerslev, K., Porto da Silva, E., Nooruzzaman, M., Amma, Y., Sasaki, Y., Mizuno, T., Miyamoto, Y., Ottaviano, L., Semenova, E., Guan, P., Zibar, D., Galili, M., Yvind, K., Morioka, T., \& Oxenløwe, L. K. (2018). Single-source chip-based frequency comb enabling extreme parallel data transmission. Nature Photonics, 12(8), 469-74. https://doi.org/10.1038/s41566-018-0205-5

\section{General rights}

Copyright and moral rights for the publications made accessible in the public portal are retained by the authors and/or other copyright owners and it is a condition of accessing publications that users recognise and abide by the legal requirements associated with these rights.

- Users may download and print one copy of any publication from the public portal for the purpose of private study or research.

- You may not further distribute the material or use it for any profit-making activity or commercial gain

- You may freely distribute the URL identifying the publication in the public portal 


\section{Single-Source Chip-based Frequency Comb Enabling Extreme Parallel Data Transmission}

Hao $\mathrm{Hu}^{1 * \dagger}$, Francesco Da $\mathrm{Ros}^{1} \dagger$, Minhao $\mathrm{Pu}^{1} \dagger$, Feihong $\mathrm{Ye}^{1}$, Kasper Ingerslev ${ }^{1}$, Edson Porto da Silva $^{1}$, Md. Nooruzzaman ${ }^{1}$, Yoshimichi Amma², Yusuke Sasaki ${ }^{2}$, Takayuki Mizuno ${ }^{3}$, Yutaka Miyamoto $^{3}$, Luisa Ottaviano ${ }^{1}$, Elizaveta Semenova ${ }^{1}$, Pengyu Guan ${ }^{1}$, Darko Zibar ${ }^{1}$, Michael Galili $^{1}$, Kresten Yvind ${ }^{1}$, Toshio Morioka ${ }^{1}$, and Leif K. Oxenløwe ${ }^{1}$

Affiliations:

${ }^{1}$ DTU Fotonik, Technical University of Denmark, Building 343, DK-2800 Lyngby, Denmark.

${ }^{2}$ Advanced Technology Laboratory, Fujikura Ltd., Sakura, Chiba, 285-8550, Japan.

${ }^{3}$ NTT Network Innovation Laboratories, NTT Corporation, Hikarinooka, Yokosuka-shi, Kanagawa, 239-0847, Japan.

*Correspondence to: huhao@fotonik.dtu.dk

† These authors contributed equally to this work.

Abstract: The Internet today transmits 100's of terabits per second, consumes $10 \%$ of all electricity worldwide ${ }^{1,2}$, and is still growing. To up-scale network capacity, massively parallel communication links with many parallel lasers are deployed. A single broadband source may substitute the parallel lasers and improve system energy-efficiency ${ }^{3,4}$. We present a frequency comb realised by a non-resonant aluminum-gallium-arsenide-on-insulator (AlGaAsOI) nanowaveguide with $66 \%$ pump-to-comb conversion efficiency, which is higher than state-of-the-art resonant comb sources. This enables unprecedented high data-rate transmission for chip-based 
sources, demonstrated using a single-mode 30-core fiber. We show that a frequency comb from our chip can carry 661 Tbit/s of data, equivalent to more than the total internet traffic today. The comb is obtained by seeding the AlGaAsOI chip with 10-GHz picosecond pulses at a low pump power (85 $\mathrm{mW})$, and this scheme is robust to temperature changes, energy-efficient and facilitates future integration with on-chip lasers or amplifiers ${ }^{5,6}$.

As worldwide data traffic continues to grow, it becomes urgent to utilise communication resources optimally, facilitating capacity growth at reduced energy consumption ${ }^{7}$. Space-division multiplexing (SDM) has been proposed as a promising solution to go beyond the looming capacity crunch $^{8-15}$. However, energy consumption is becoming another limit for further scalingup of large-capacity transmission systems. SDM schemes are anticipated to be combined with wavelength-division multiplexing (WDM), currently requiring hundreds of individual lasers at different wavelengths and each laser needing cooling to be stabilised ${ }^{14}$. A single laser seeded frequency comb is attractive, since it may replace massive numbers of parallel lasers using individual comb lines, with much lower energy consumption and smaller space occupation ${ }^{11}$. Recently, various chip-based frequency comb sources have been demonstrated enabling WDM data transmission, such as mode-locked laser based 12-Tbit/s transmission ${ }^{16}$. However, modelocked lasers tend to have a limited bandwidth by themselves. Frequency combs have also been demonstrated by soliton comb generation in micro-resonators and even used for up to 50-Tbit/s data transmission ${ }^{17,18}$. However, this technique is challenged by a low pump-to-comb conversion efficiency, with often only $<1 \%$ of the pump being converted to the generated frequency comb ${ }^{17}$. In addition, micro-resonators inherently rely on a delicate balance between Kerr nonlinearity, 
dispersion, and thermal resonance shifts in order to generate soliton frequency combs ${ }^{19}$. This introduces a need for active stabilization to ensure long-term stability ${ }^{20}$.

In contrast, broadband frequency combs can also be achieved by spectral broadening of a narrow-band frequency comb $\mathrm{b}^{3,4}$, which can have a high pump-to-comb conversion efficiency. Furthermore, the non-resonant nature of the scheme makes it more resilient to temperature changes and facilitates long-term stability. Frequency comb broadening based on highly nonlinear fibers (HNLFs) has been used for optical communications ${ }^{3,4,15}$. However, a long interaction length (typically tens to hundreds of meters) is required for HNLFs to achieve good conversion, which renders HNLFs too bulky for integration on a chip. Comb broadening has also been demonstrated in integrated waveguides of various material platforms ${ }^{21,22}$, with the used of femtosecond pump pulses with high peak powers and typically with low repetition rates ( $<1$ GHz). However, a high comb line spacing between $10 \mathrm{GHz}$ and $50 \mathrm{GHz}$ is desired for optical communications, which requires pump pulses with a similar repetition rate. The peak-to-average power ratio of such pulses is much lower than that of fs-pulses at low repetition rates, making it very challenging to realise efficient on-chip frequency comb broadening at high rates.

In this paper, we explore chip-based frequency combs as single-source emitters for optical communications, and in particular investigate how much data can be carried on the light from a single chip. We encode decorrelated data on 2400 parallel SDM/WDM channels with 16-state quadrature amplitude modulation (16-QAM) in combination with Nyquist optical time division multiplexing (TDM) and polarization division multiplexing (PDM). In this way, six physical dimensions (amplitude, phase, time, frequency, polarization and space) are used for data modulation and multiplexing in order to boost the total data rate. We demonstrate the transmission of 661 Tbit/s data carried on the broadened frequency comb from a single highly- 
nonlinear AlGaAs-on-insulator (AlGaAsOI) nano-waveguide ${ }^{23,24}$, which is pumped by $10-\mathrm{GHz}$ picosecond pulses with an average launched power of only $85 \mathrm{~mW}$. The non-resonant AlGaAsOI waveguide is operated without a temperature controller or a feedback loop for stabilization. The low loss and high nonlinearity characteristics of the AlGaAsOI waveguide enables efficient frequency comb broadening, and the high pump-to-comb conversion efficiency allows for sufficient power per comb line at the chip output.

AlGaAsOI has recently emerged as an ultra-efficient nonlinear platform, since it combines a high intrinsic material nonlinearity with a large refractive index contrast between the nonlinear medium and the cladding materials ${ }^{25}$. The bandgap of AlGaAs can be engineered by changing the $\mathrm{Al}$ concentration to avoid two-photon absorption (TPA) at telecom wavelengths ${ }^{26}$. All of this renders AlGaAsOI a good platform for self-phase modulation (SPM) based optical frequency comb spectral broadening (Fig. 1a). Owing to the large index contrast of this structure, light can be strongly confined in a sub-micron waveguide core (Fig. 1b), which enhances the light-matter interaction and results in an ultra-high nonlinear parameter $(\gamma)$ of $\sim 660 \mathrm{~W}^{-1} \mathrm{~m}^{-1}$ for an AlGaAsOI nano-waveguide ${ }^{23}$. Compared with a typical HNLF, the AlGaAsOI nano-waveguide exhibits about three orders of magnitude higher figure of merit, which is defined by the ratio of the maximal achievable nonlinear phase shift and the nonlinear medium length (Supplementary material). In addition, the waveguide dispersion dominates over the material dispersion for subwavelength-sized waveguides and therefore the group velocity dispersion (GVD) can be engineered to be anomalous at telecom wavelengths (Fig. S1), which is desired to achieve efficient SPM induced frequency comb broadening.

The frequency spacing between the comb lines is determined by the repetition rate of the seed pulses (Fig. 2), which is locked to the 10-GHz clock from the transmitter and can be 
controlled with high accuracy at $\mathrm{Hz}$ level (Fig. S5). The generated frequencies can be stably aligned to the ITU (International Telecommunication Union) grid by fine tuning the seed laser center frequency $\mathrm{y}^{27}$. The on-chip frequency comb broadening exhibits a very high pump-to-comb conversion efficiency of $66 \%$, thus offering a more power-efficient solution than the soliton comb generation based on resonant enhancement ${ }^{19}$. The laser linewidth is another key property of a frequency comb, as a narrow linewidth is required to be able to modulate both amplitude and phase of the optical signal. The linewidths of the broadened frequency comb at the wavelengths of $1529.97 \mathrm{~nm}$ and $1560.09 \mathrm{~nm}$ are measured to be $40 \mathrm{kHz}$ at both wavelengths (Fig. S2), which are the same as that of the seed mode-locked laser and well below the requirement for the 16QAM modulation at $10 \mathrm{GBd}^{28}$. Thus, we did not observe linewidth degradation due to the comb broadening within the measured wavelength range, as expected given the correlated phase of the seed laser ${ }^{29}$.

To investigate how much data the generated frequency comb can sustain, it is tested in a challenging scenario of multidimensional modulation and multiplexing. Fig. 3a shows the concept of frequency comb source based six-dimensional data modulation and multiplexing for large-capacity optical communications. Amplitude and phase are used for data modulation; time, polarization, wavelength and space are used for multiplexing, i.e. as independent dimensions where each adds additional data channels. The generated comb lines serve as the WDM source for multiple wavelength channels. Fig. 3b shows the scheme of the large-capacity sixdimensional data transmission using a single source chip-based frequency comb. The broadened frequency comb at the output of the AlGaAsOI photonic chip is amplitude equalised using a wavelength selective switch (WSS) and then split into 30 copies. Each of the frequency comb copies is further separated in wavelength using WDM demutiplexers and act as light sources for 
80 WDM channels. Each WDM channel is modulated by 10 GBaud 16-QAM, encoding 4 bits on each pulse, multiplexed in time by a factor of 4 , and multiplexed by orthogonal polarizations using a polarization beam combiner (PBC). The data rate of each modulated TDM-PDM-16QAM channel is $10 \mathrm{Gbit} / \mathrm{s} \times 4(16$ QAM modulation $) \times 4(\mathrm{TDM}) \times 2(\mathrm{PDM})=320 \mathrm{Gbit} / \mathrm{s}$. These channels are multiplexed in wavelength $(\times 80)$ by a WDM multiplexer and finally launched into the 30-core fiber through a fan-in device, resulting in 2400 parallel data channels with an aggregate gross data rate of $768 \mathrm{Tbit} / \mathrm{s}(320 \mathrm{Gbit} / \mathrm{s} \times 2400)$.

The heterogeneous single-mode 30-core fiber uses a trench-assisted refractive index structure and an inter-core phase mismatching by using four types of cores to reduce mode coupling between adjacent cores ${ }^{12}$, as shown in Fig. S10. As a result, a high-density core arrangement with inter-core crosstalk below $-50 \mathrm{~dB}$ after $9.6 \mathrm{~km}$ is achieved. This allows for direct reception of the SDM channels without additional multiple-input-multiple-output (MIMO) processing. The 30 cores are arranged within a cladding diameter of $228 \mu \mathrm{m}$, which is less than the $250 \mu \mathrm{m}$ constraint to maintain mechanical stability comparable to that of standard single-mode fibers.

The schematic of the implemented experimental setup is shown in Fig. 4a. The single source laser in the transmitter is a mode-locked laser, which generates $10-\mathrm{GHz}$ pulses with a full width at half maximum (FWHM) of 1.5 ps. The pulses are amplified and launched into the 5-mm-long AlGaAsOI nano-waveguide, with an average launched power of only $85 \mathrm{~mW}$ (peak power of 5.6 W), in order to achieve SPM based frequency comb broadening. The average power of the broadened frequency comb at the output of the chip is $\sim 13 \mathrm{~mW}$, i.e. 11 decibels relative to $1 \mathrm{~mW}$ (dBm). This corresponds to an average power of $\sim 32 \mu \mathrm{W}$ (i.e. $-15 \mathrm{dBm}$ ) per comb line for $\sim 400$ comb lines within the telecom $\mathrm{C}$ band. The center part $(5 \mathrm{~nm})$ of the frequency comb has a large 
power variation, resulting in optical signal to noise ratio (OSNR) degradation for the low-power part $^{2}$. Therefore, the center part from $1540.73 \mathrm{~nm}$ to $1545.87 \mathrm{~nm}$ is replaced with the original spectrum from the mode-locked laser by passing through the other path of the first WSS, resulting in a flat and high-quality frequency comb (Fig. 4b). More details about the experimental setup are given in the method section.

To properly evaluate the chip-based single source for carrying multi-100 Tbit/s, all comb lines are simultaneously data modulated and then launched into the 30-core fiber. Using this approach, we treat all the 2400 SDM/WDM channels a like and we sequentially characterise each of them. To evaluate the quality of the transmitted data, bit error rates (BER) are measured after transmission for all the 80 WDM channels over the 30 spatial channels (Fig. 4d). All the 80 WDM channels are below forward error correction (FEC) limits, with 58 WDM channels below the soft-decision FEC limit (20\% overhead) and 22 WDM channels below the hard-decision FEC limit (7\% overhead). For the data channels with a BER below a certain FEC limit, a BER $<10^{-15}$ can be achieved after the error correction ${ }^{30}$, which is considered as error-free performance. Therefore, the BER measurements confirm the successful transmission of all the 2400 channels with a net rate of $661 \mathrm{Tbit} / \mathrm{s}$ after FEC overhead subtraction. This achievement is mainly enabled by the high nonlinearity and low linear and nonlinear loss of the AlGaAsOI nano-waveguide, which offers sufficient frequency comb output power with a low pump power. The low crosstalk of the 30-core fiber facilitates low-complexity reception of the signal without multiple-inputmultiple-output (MIMO) processing. The measured BERs of all channels depend on wavelength, resulting from the spectral shape of the broadened frequency comb and the increased noise at the edge of the C-band. 
To investigate the power-efficiency of using the chip-based frequency comb, we compare it with parallel WDM lasers. The power savings of using the AlGaAsOI chip based frequency comb source scales with the number of WDM channels. The power consumption of using the single-source frequency comb is only $1 / 4$ to $1 / 15$ of the power consumption of using parallel WDM lasers for 80 to 400 channels (number of lasers) (Supplementary materials).

We have presented a photonic chip based frequency comb with a sufficient comb output power to support several hundred Tbit/s optical data. This is the highest data rate using a chipbased frequency comb source, which may potentially replace hundreds of parallel lasers with reduced power consumption. With further optimization of the dispersion of the AlGaAsOI waveguide, the shape of the broadened frequency comb could become smoother and the OSNR of the low-power part could be increased. This may allow for higher-order modulation format and higher transmission rates. With the low pump power requirement, this scheme has potential $\underline{\text { for realization of a fully integrated frequency comb source including chip-based pump lasers }^{5}}$ and amplifiers ${ }^{6}$. Other components required for equalization and splitting, such as wavelength selective switches and WDM demultiplexers, also have integration solutions ${ }^{31,32}$. Moreover, the comb source is scalable to even broader bandwidths such as S, C and L bands (Supplementary materials), and is thus promising for photonic chip-based single source transmission beyond Petabit/s. 


\section{References}

1. Cisco “Cisco Visual Networking Index: Forecast and Methodology, 2016-2021” (2016) http://www.cisco.com/c/en/us/solutions/collateral/service-provider/visual-networking-indexvni/complete-white-paper-c11-481360.html

2. Mills, M. The Cloud Begins With Coal. Digital Power Group. (2013) https://www.techpundit.com/wp-content/uploads/2013/07/Cloud_Begins_With_Coal.pdf

3. Hillerkuss, D. et al. 26 Tbit s-1 line-rate super-channel transmission utilizing all-optical fast Fourier transform processing. Nature Photonics 5, 364-371 (2011).

4. Ataie, V. et al. Ultrahigh Count Coherent WDM Channels Transmission Using Optical Parametric Comb-Based Frequency Synthesizer. J. Lightwave Technol. 33, 694-699 (2015).

5. S. Uvin, S. Keyvaninia, F. Lelarge, G. H. Duan, B. Kuyken, and G. Roelkens, Narrow line width frequency comb based on an injection-locked III-V-on-silicon mode-locked laser. Opt. Express 24, 5277-5286 (2016).

6. Akiyama, T. et al. An ultrawide-band semiconductor optical amplifier having an extremely high penalty-free output power of $23 \mathrm{dBm}$ achieved with quantum dots. IEEE Photonics Technology Letters 17, 1614-1616 (2005).

7. Essiambre, R.-J., Kramer, G., Winzer, P. J., Foschini, G. J. \& Goebel, B. Capacity Limits of Optical Fiber Networks. J. Lightwave Technol. 28, 662-701 (2010).

8. Morioka, T. New generation optical infrastructure technologies: "EXAT initiative” towards 2020 and beyond, OptoElectronics and Communications Conference (OECC) 2009, FT4 (2009).

9. Richardson, D. J. Filling the Light Pipe. Science 330, 327-328 (2010).

10. Bozinovic, N. et al. Terabit-Scale Orbital Angular Momentum Mode Division Multiplexing in Fibers. Science 340, 1545-1548 (2013). 
11. Hu, H. et al. Single-Source AlGaAs Frequency Comb Transmitter for 661 Tbit/s Data Transmission in a 30-core Fiber. in Conference on Lasers and Electro-Optics (CLEO): 2016 Postdeadline Paper Digest, paper JTh4C.1 (2016).

12. Matsuo, S. et al. High-Spatial-Multiplicity Multicore Fibers for Future Dense Space-DivisionMultiplexing Systems. J. Lightwave Technol. 34, 1464-1475 (2016).

13. Mizuno, T. et al. Long-Haul Dense Space-Division Multiplexed Transmission Over Low-Crosstalk Heterogeneous 32-Core Transmission Line Using a Partial Recirculating Loop System. J. Lightwave Technol. 35, 488-498 (2017).

14. Kobayashi, T. et al. 1-Pb/s (32 SDM/46 WDM/768 Gb/s) C-band Dense SDM Transmission over 205.6-km of Single-mode Heterogeneous Multi-core Fiber using 96-Gbaud PDM-16QAM Channels. in Optical Fiber Communication Conference Postdeadline Papers (2017), paper Th5B.1 (Optical Society of America, 2017).

15. Puttnam, B. J. et al. $2.15 \mathrm{~Pb} / \mathrm{s}$ transmission using a 22 core homogeneous single-mode multi-core fiber and wideband optical comb. in 2015 European Conference on Optical Communication (ECOC) paper PDP.3.1 (2015).

16. Kemal, J. N. et al. 32QAM WDM Transmission Using a Quantum-Dash Passively Mode-Locked Laser with Resonant Feedback. in Optical Fiber Communication Conference Postdeadline Papers (2017), paper Th5C.3 (Optical Society of America, 2017).

17. Marin-Palomo, P. et al., Microresonator-based solitons for massively parallel coherent optical communications. Nature. 546, 274-279 (2017).

18. Fülöp, A. et al. Long-haul coherent communications using microresonator-based frequency combs. Opt. Express 25, 26678-26688 (2017).

19. Brasch, V. et al. Photonic chip-based optical frequency comb using soliton Cherenkov radiation. Science 351, 357-360 (2016).

20. Huang, S.-W. et al. A broadband chip-scale optical frequency synthesizer at $2.7 \times 10^{-16}$ relative uncertainty. Science Advances 2, e1501489 (2016). 
21. Halir, R. et al. Ultrabroadband supercontinuum generation in a CMOS-compatible platform. Opt. Lett. 37, 1685-1687 (2012).

22. Kuyken, B. et al. An octave-spanning mid-infrared frequency comb generated in a silicon nanophotonic wire waveguide. Nat. Commun. 6, 6310 (2015).

23. Pu, M., Ottaviano, L., Semenova, E. \& Yvind, K. Efficient frequency comb generation in AlGaAson-insulator. Optica, 3, 823-826 (2016).

24. Hu, H. et al. Supercontinuum comb sources for broadband communications based on AlGaAs-oninsulator. in Proceedings of SPIE (Vol. 10088). [100880C] SPIE - International Society for Optical Engineering (International Society for Optics and Photonics, 2017).

25. Ottaviano, L., Pu, M., Semenova, E. \& Yvind, K. Low-loss high-confinement waveguides and microring resonators in AlGaAs-on-insulator. Opt. Lett. 41, 3996-3999 (2016).

26. Wathen, J. J. et al. Efficient continuous-wave four-wave mixing in bandgap-engineered AlGaAs waveguides. Opt. Lett. 39, 3161-3164 (2014).

27. Mori, K., Sato, K., Takara, H. \& Ohara, T. Supercontinuum lightwave source generating $50 \mathrm{GHz}$ spaced optical ITU grid seamlessly over S-, C- and L-bands. Electronics Letters 39, 544-546 (2003).

28. Seimetz, M. High-Order Modulation for Optical Fiber Transmission. (Springer, 2009).

29. Tong, Z. et al. Spectral linewidth preservation in parametric frequency combs seeded by dual pumps. Opt. Express, 20, 17610-17619 (2012).

30. Digital Video Broadcasting Second generation (DVB S2), ETSI EN 302 307, V1.2.1 (2009).

31. Vettese, D. Liquid crystal on silicon. Nature Photonics 4, 752 (2010).

32. Pathak, S. et al. Optimized Silicon AWG With Flattened Spectral Response Using an MMI Aperture. J. Lightwave Technol. 31, 87-93 (2013).

33. Dar, R. et al. Impact of WDM Channel Correlations on Nonlinear Transmission. in ECOC 2016; 42nd European Conference on Optical Communication, 482-484 (2016). 


\section{Acknowledgments:}

This work was funded by the SPOC research center of excellence (DNRF123), the NATEC Villum center of excellence and the EU-Japan coordinated R\&D project on "Scalable And Flexible optical Architecture for Reconfigurable Infrastructure (SAFARI)" commissioned by the MIC, Japan and EC Horizon 2020. H.H. acknowledges P.-Y. Bony for help with the linewidth measurement.

\section{Corresponding author}

Correspondence to Hao Hu (huhao@fotonik.dtu.dk)

\section{Author contributions:}

H.H. conceived and designed the experiments. F.D.R. and E.P.S. conducted digital signal processing for the transmitted data. M.P. designed the AlGaAs device. H.H., F.D.R., F.Y., K.I. and M.N performed the transmission experiment. H.H., F.D.R and M.P. analyzed the data. M.P., L.O., E.S. and K.Y. fabricated the AlGaAs device. H.H. and M.P. characterized the AlGaAs device. F.Y., Y.A., Y.S. and T.M. designed the multi-core fiber. Y.A. and Y.S. fabricated the multi-core fiber. T.M., Y.M., P.G., D.Z., M.G., L.K.O. and T.M. contributed to the experiment. H.H. and L.K.O. wrote the manuscript and all the coauthors contributed to the writing. Y.M., K.Y., T.M. and L.K.O. supervised the projects.

\section{Methods}


Design and fabrication of the AlGaAsOI nano-waveguides. We have developed an AlGaAson-insulator (AlGaAsOI) platform ${ }^{23}$, where a thin $\mathrm{Al}_{x} \mathrm{Ga}_{1-x} \mathrm{As}$ layer on top of a low index insulator layer resides on a semiconductor substrate as shown in Fig. 1b. Wafer bonding and substrate removal are used to realize AlGaAsOI wafers ${ }^{25}$. Electron beam lithography (EBL, JEOL JBX-9500FS) was used to define the nano-waveguide pattern in the electron beam resist hydrogen silsesquioxane (HSQ, Dow Corning FOX-15). The nano-waveguide pattern was then transferred into the AlGaAs layer using a boron trichloride $\left(\mathrm{BCl}_{3}\right)$-based dry etching process in an inductive coupled plasma reactive ion etching (ICP-RIE) machine. As the refractive index of HSQ is relatively low (similar to $\mathrm{SiO}_{2}$ ), it was kept on top of the AlGaAs device pattern. Finally, clad in a 3- $\mu \mathrm{m}$ thick silica layer using plasma-enhanced chemical vapour deposition (PECVD). Owing to the large index contrast ( $\sim 5 \%)$ between AlGaAs and silica, light can be confined in the sub-micron waveguide core. The strong light confinement not only effectively enhances the device nonlinearity but also enable efficient dispersion engineering. The performance of nanowaveguide devices is also limited by the linear loss induced by light scattering due to surface roughness. To reduce the surface roughness, all the fabrication processes have been optimized. High quality epitaxial material growth and substrate removal are required to ensure smoothness for top and bottom waveguide surfaces, while high-quality electron-beam lithography and dry etching processes ensure a small roughness on the waveguide sidewall surfaces (Fig. 1b). The propagation loss of the fabricated AlGaAsOI waveguide is about $1.5 \mathrm{~dB} / \mathrm{cm}^{23}$. The chip was cleaved to form the input and output facets where nano-tapers enabled efficient chip-to-fibre coupling for characterization. Tapered fibers are used at both facets and the coupling loss is $\sim 3 \mathrm{~dB} /$ facet. 
Data modulation and multiplexing. In our experiment, the broadened frequency comb with $10 \mathrm{GHz}$ spacing is modulated with $10 \mathrm{GBaud}$ 16-QAM in a standard I/Q modulator driven by a 60 GSample/s arbitrary waveform generator (AWG) with $20 \mathrm{GHz}$ of analog bandwidth. The modulated 10 GBaud 16-QAM signal is optical time division multiplexed (OTDM) by a factor of four to 40 GBaud based on time-domain pulse interleaving in a passive fiber-delay multiplexer $(\mathrm{MUX} \times 4)$. A delay-and-add polarization multiplexing emulator is used to generate PDM signals, corresponding to the data rate of $320 \mathrm{Gbit} / \mathrm{s}$ per wavelength channel (40 GBaud serial rates, 4 bits per symbol by 16-QAM modulation and two polarizations). In addition, optical Nyquist filtering was used to strongly confine the spectrum of each WDM channel, resulting in a rectangular spectrum in the frequency domain and sinc-function waveforms in the time domain with the duty cycle of 25\% (Fig. 3a). Although the sinc-shaped pulses strongly overlap with neighboring pulses, inter-symbol interference (ISI) is minimized by having one sinc-pulse’s zero-crossing coincide with its neighbor’s peak.

To generate a WDM signal with $50 \mathrm{GHz}$ spacing, the broadened frequency comb is spectrally sliced into odd and even channels and separated into two paths using a second WSS. The delay difference between the two paths is $7.5 \mathrm{~ns}$ in order to de-correlate the odd and even channels. This is a laboratory emulation of a real WDM transmission system, which can be used to characterize the transmission performance ${ }^{33}$. All the WDM channels are modulated by one I/Q modulator before the splitting and de-correlation in the lab experiment. The I/Q modulator has a maximum input power of $32 \mathrm{~mW}$ (i.e. $15 \mathrm{dBm}$ ) and an insertion loss of $\sim 17 \mathrm{~dB}$. The insertion loss is mainly limited by operating the I/Q modulator close to the linear regime. Therefore, the average input power for each of $\sim 400$ comb lines is limited to be $-11 \mathrm{dBm}$ and the average output power for each comb line is only $-28 \mathrm{dBm}$ at the output of the modulator. The modulated WDM 
channels are amplified by an erbium-doped fiber amplifier (EDFA), however, the signal OSNR will be significantly degraded with the low power at the input of the EDFA. Since the average power $(-28 \mathrm{dBm})$ for each comb line at the output of the modulator is much lower than the average power $(-15 \mathrm{dBm})$ for each comb line at the output of the AlGaAs chip, the signal OSNR is mainly limited by the noise generated in the EDFA at the output of the modulator. In real WDM systems, the WDM channels are modulated independently with parallel modulators, and the input power of the modulator for each WDM channel is significantly higher. Therefore, the real-world transmission performance is underestimated in the lab experiment, limited by available lab equipment, and better transmission performance is expected for real WDM systems.

The second WSS in the experimental setup is programmed for rectangular filtering with a bandwidth of $40 \mathrm{GHz}$ and $50 \mathrm{GHz}$ spacing (compatible with the ITU standard), in order to generate 40 GBaud Nyquist TDM-PDM-16-QAM signals for all the WDM channels. A $10 \mathrm{GHz}$ guard band was inserted in between the WDM channels in order to minimize inter-channel crosstalk. The odd and even WDM channels are recombined using a third WSS. As a result, 80 WDM channels between $1529.97 \mathrm{~nm}$ and $1562.92 \mathrm{~nm}$ are generated to reach a data rate of 25.6 Tbit/s (Fig. 4c). 30 SDM channels are fully de-correlated to each other, with at least $2.5 \mathrm{~ns}$ between SDM channels. These SDM channels are multiplexed by a 3D-waveguide based fan-in device, and then launched into the 9.6-km heterogeneous single-mode 30-core fiber. The launched power for each core is between $14-18 \mathrm{dBm}$ accounting for loss-variations in the fanin/fan-out from $5 \mathrm{~dB}$ to $8 \mathrm{~dB}$.

Coherent Receiver. After the 30-core transmission, the 30 spatial channels are demultiplexed using another 3D-waveguide based fan-out device. A tunable bandpass filter with a bandwidth of $50 \mathrm{GHz}$ is used to select each WDM channel. The selected WDM channel is detected with a 
dual-polarization coherent receiver, consisting of a polarization-diversity 90-degree hybrid, a local oscillator (LO) and four balanced detectors, followed by a digital sampling oscilloscope (DSO, 80 GSamples/s, $33 \mathrm{GHz}$ bandwidth). The waveforms were processed from 0.5 MSamples by offline digital signal processing (DSP) including adaptive time-domain equalization using constant and multi-modulus algorithms, OTDM demultiplexing, carrier recovery by decisiondirected phase locked loop, demapping and bit error rate (BER) counting. Carrier recovery, demapping and BER counting were performed on each OTDM tributary separately.

The data that support the plots within this paper and other findings of this study are available from the corresponding author upon reasonable request. 

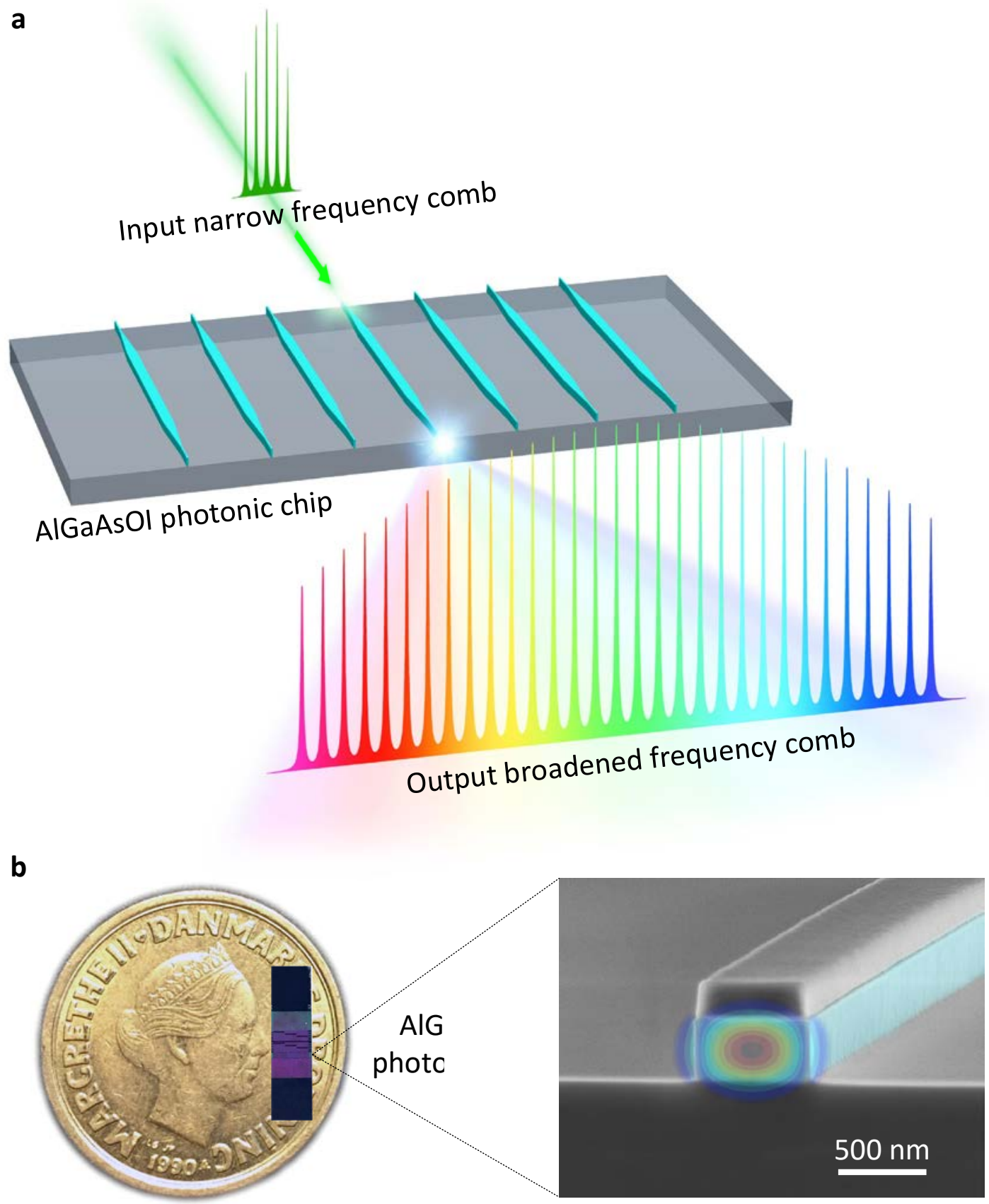

Fig. 1. AIGaAsOI photonic chip. (a) A narrow-band frequency comb is spectrally broadened to a broadband frequency comb through self-phase modulation by passing through an AlGaAsOI nano-waveguide. (b) The AlGaAsOI photonic chip is smaller than a coin, but can accommodate hundreds of nano-waveguides. The scanning electron microscopy image shows the cross section of the nano-waveguide with cross-section dimensions of $280 \mathrm{~nm}$ $\times 600 \mathrm{~nm}$, indicating strong light confinement (artificially added the simulated field distribution for the fundamental TE mode). 


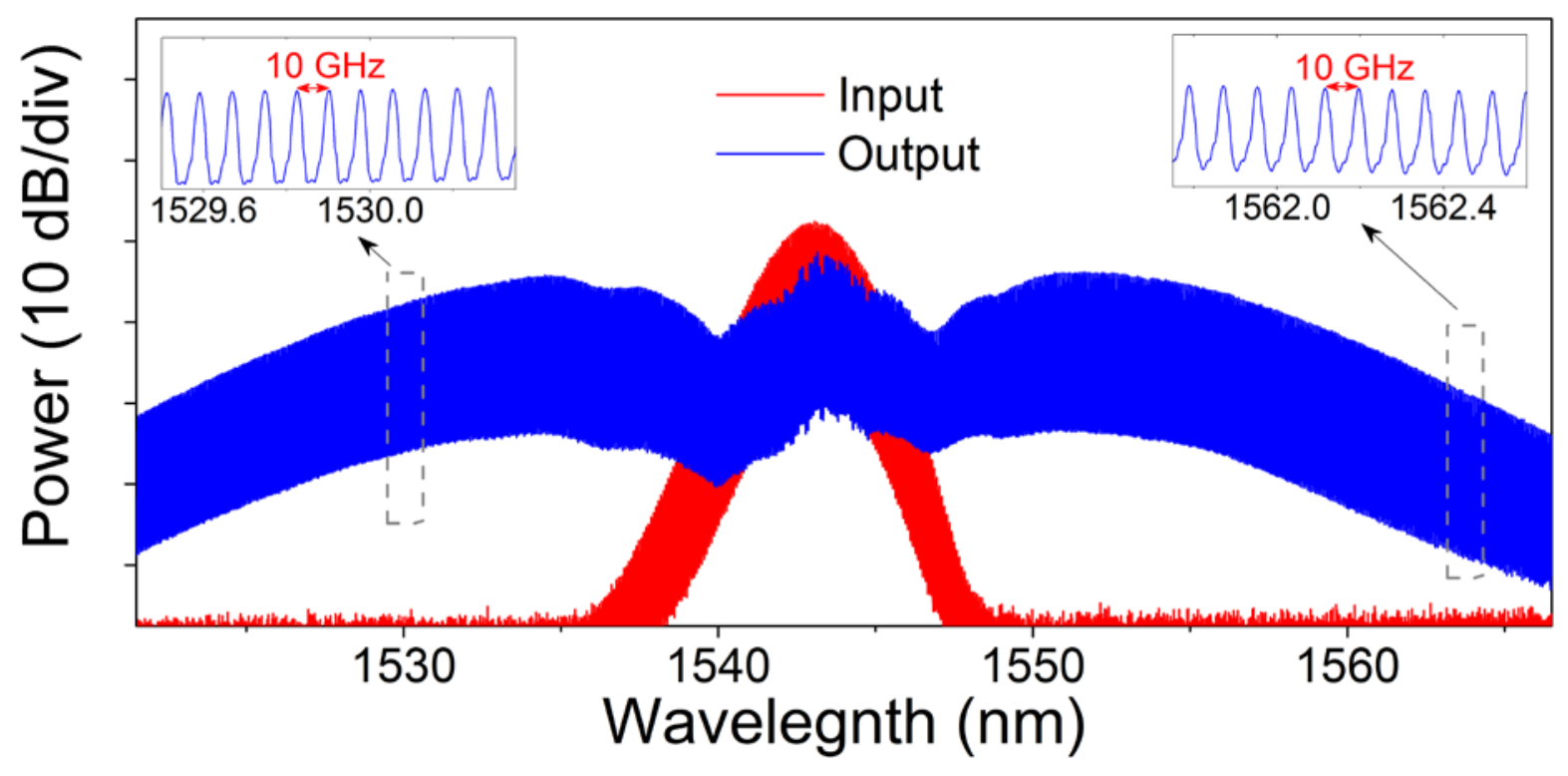

Fig. 2. Frequency comb broadening in an AIGaAsOI nano-waveguide. A 10-GHz 1.5-ps pulse generated from a mode-locked laser is launched into the AlGaAsOI nano-waveguide, with an average launched power of only $85 \mathrm{~mW}$. The input frequency comb (20-dB bandwidth of $6.4 \mathrm{~nm})$ is spectrally broadened by SPM to a 20-dB bandwidth of $\sim 44 \mathrm{~nm}$, covering more than the telecom C band. All comb lines are equidistantly spaced by $10 \mathrm{GHz}$, exactly following the repetition rate of the seed laser. 
a
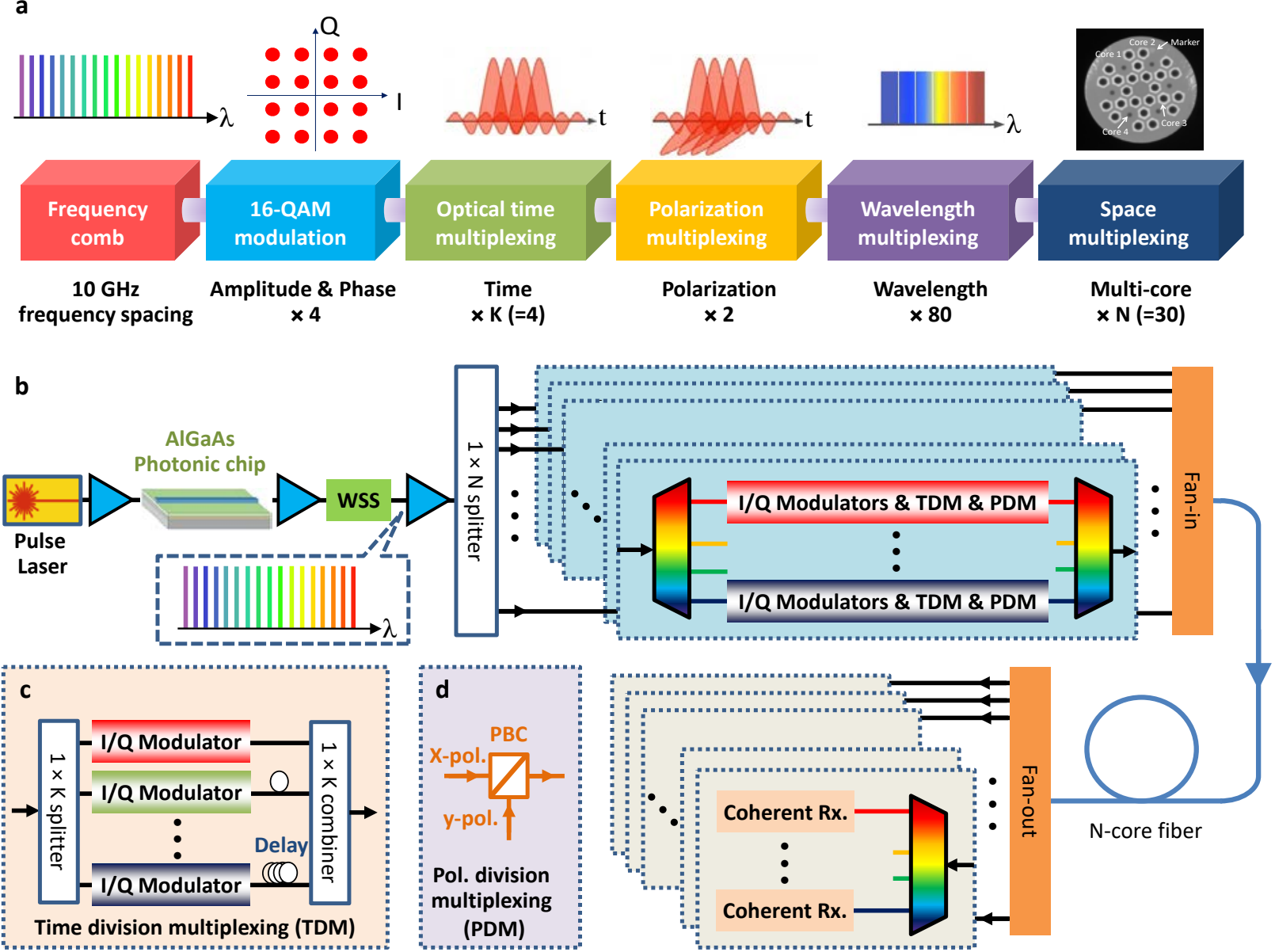

Fig. 3. Generation and transmission of multi-100 Tbit/s data carried by the AIGaAsOI SPM-based frequency

comb. a, Concept of frequency comb source based six-dimensional data modulation and multiplexing. The chipbased frequency comb is used as WDM sources. Amplitude and phase are modulated with 16 states in the complex plane of the optical field, corresponding to four bits of information per pulse. Optical pulses are temporally interleaved and then filtered in the frequency domain using a rectangular spectral shape, resulting in sinc-shaped waveforms in the time domain. Data is multiplexed in two orthogonal polarizations. 80 WDM channels are multiplexed and launched into a multi-core fiber. b, Implementation scheme of large-capacity 6-dimensional data transmission using chip-based frequency comb source. c, Time division multiplexing (TDM) based on time-domain pulse interleaving in a passive fiber-delay multiplexer. d, Polarization division multiplexing (PDM) by combining two orthogonal polarizations (x-pol. and y-pol.) using a polarization beam combiner (PBC). 
a

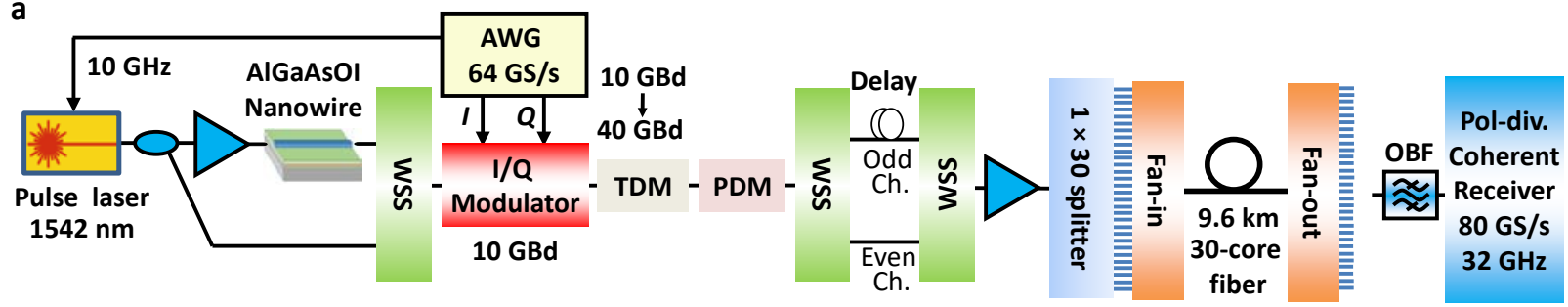

b

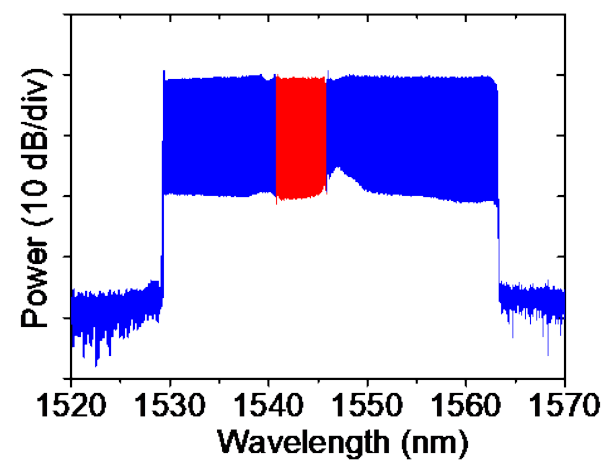

C

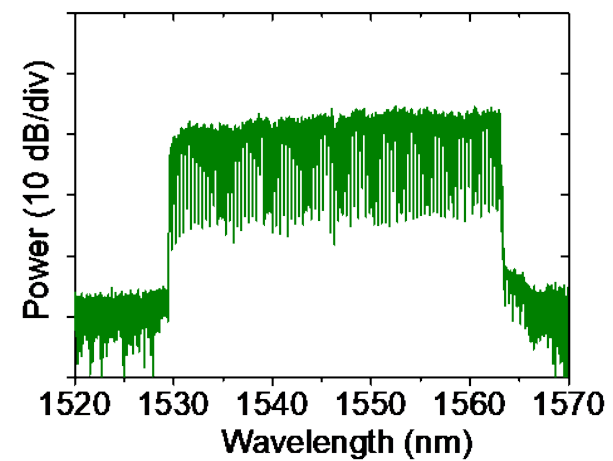

d

WDM Channel (\#)

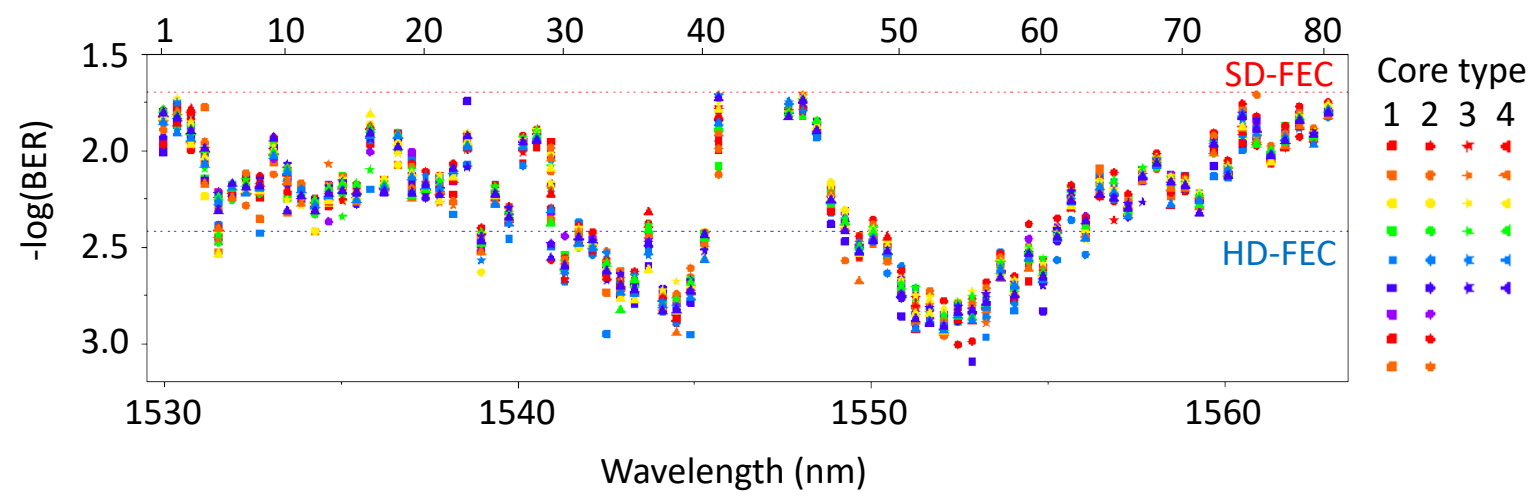

Fig. 4. $661 \mathrm{Tbit} / \mathrm{s}$ data transmission using chip-based frequency comb source. a, Schematic of the implemented experimental setup for the transmission of 661 Tbit/s with 2400 parallel channels over a 30-core fiber. b, The generated frequency comb is amplitude equalized (blue) in a wavelength selective switch and the low-quality part in the center is replaced by the original spectrum (red) from the seed laser, resulting in a flat and high-quality frequency comb, which is subsequently data modulated and transmitted. c, The comb spectrum with data after $9.6 \mathrm{~km}$ 30-core fiber transmission and amplification. d, BER measurements for the $80 \mathrm{WDM}$ channels over the 30 spatial channels. All the 2400 WDM/SDM channels are below the FEC limits, which can achieve a BER $<10^{-15}$ after the error correction. A few center channels have BERs above the FEC limits due to degraded OSNR, not shown in the figure. 